Electromagnetic Brain Imaging 
Kensuke Sekihara $\cdot$ Srikantan S. Nagarajan

\section{Electromagnetic Brain Imaging}

A Bayesian Perspective

黛 Springer 
Kensuke Sekihara

Department of Systems Design and Engineering

Tokyo Metropolitan University

Tokyo

Japan
Srikantan S. Nagarajan

Department of Radiology and Biomedical

University of California

San Francisco, CA

USA

ISBN 978-3-319-14946-2

ISBN 978-3-319-14947-9 (eBook)

DOI 10.1007/978-3-319-14947-9

Library of Congress Control Number: 2014959853

Springer Cham Heidelberg New York Dordrecht London

(C) Springer International Publishing Switzerland 2015

This work is subject to copyright. All rights are reserved by the Publisher, whether the whole or part of the material is concerned, specifically the rights of translation, reprinting, reuse of illustrations, recitation, broadcasting, reproduction on microfilms or in any other physical way, and transmission or information storage and retrieval, electronic adaptation, computer software, or by similar or dissimilar methodology now known or hereafter developed.

The use of general descriptive names, registered names, trademarks, service marks, etc. in this publication does not imply, even in the absence of a specific statement, that such names are exempt from the relevant protective laws and regulations and therefore free for general use.

The publisher, the authors and the editors are safe to assume that the advice and information in this book are believed to be true and accurate at the date of publication. Neither the publisher nor the authors or the editors give a warranty, express or implied, with respect to the material contained herein or for any errors or omissions that may have been made.

Printed on acid-free paper

Springer International Publishing AG Switzerland is part of Springer Science+Business Media (www.springer.com) 


\section{Preface}

Neuronal activities in a human brain generate coherent synaptic and intracellular currents in cortical columns, which generate electric potentials on the scalp surface and magnetic fields outside the head. Electroencephalography (EEG) measures these potentials and magnetoencephalography (MEG) measures these magnetic fields to obtain information on the state of the brain. The class of methodologies that reconstruct and visualize the neuronal activities based on MEG/EEG sensor measurements is referred to as the electromagnetic brain imaging.

In the past two decades there have been significant advances in signal processing and source reconstruction algorithms used in electromagnetic brain imaging. However, electromagnetic brain imaging can still be considered a young field. This is primarily due to the complexity associated with the electrophysiological brain activity underlying the signals. Also, it is true that applying the electromagnetic brain imaging with confidence requires an understanding of the relevant mathematics, physics, biology, and engineering as well as a broad perspective on human brain science. Due to its interdisciplinary nature, such a broad knowledge base takes years to acquire.

This book is intended to provide a coherent introduction to the body of mainstream algorithms used in electromagnetic brain imaging, with specific emphasis on novel Bayesian algorithms that we have developed. It is intended as a graduate level textbook with the goal of helping readers more easily understand the literature in biomedical engineering and in related fields, and be ready to pursue research in either the engineering or the neuroscientific aspects of electromagnetic brain imaging. We hope that this textbook will not only appeal to graduate students but all scientists and engineers engaged in research on electromagnetic brain imaging.

This book begins with an introductory overview of electromagnetic brain imaging in Chap. 1 and then discusses dominant algorithms that are used in electromagnetic brain imaging in Chaps. 2-4. Minimum-norm-based methods, which are classic algorithms and still widely used in this field, are described in Chap. 2, but with a Bayesian perspective in mind. Chapter 3 presents a concise review of adaptive beamformers, which have become a standard tool for analyzing brain 
spontaneous activity such as resting-state MEG data, and we also include a Bayesian perspective on adaptive beamformers.

Chapters 4-6 review Bayesian algorithms for electromagnetic brain imaging that we have developed in the past decade. Chapter 4 presents a novel Bayesian algorithm, called Champagne, which has been developed by our group. Since we believe that the Champagne algorithm is a powerful next-generation imaging algorithm, the derivation of the algorithm is presented in detail. Chapter 5 presents Bayesian factor analysis, which is a group of versatile algorithms used for denoising, interference suppression, and source localization. Chapter 6 describes a unified theoretical Bayesian framework, which provides insightful perspective into various source imaging methods and reveals similarities and equivalences between methods that appear to be very different.

Chapters 7-9 deal with newer topics that are currently in vogue in electromagnetic brain imaging - functional connectivity, causality, and cross-frequency coupling analyses. Chapter 7 reviews functional connectivity analysis using imaginary coherence. Chapter 8 provides a review of several directional measures that can detect causal coupling of brain activities. Chapter 9 presents novel empirical results showing that the phase-amplitude coupling can be detected using MEG source-space analysis, and demonstrates that the electromagnetic brain imaging holds great potential in elucidating the mechanisms of brain information processing. This chapter was contributed by Eiichi Okumura and Takashi Asakawa. The first two chapters in the Appendix provide concise explanations of bioelectromagnetic forward modeling and basics of Bayesian inference, and the third chapter provides supplementary mathematical arguments. These chapters are included for the reader's convenience.

Many people have made valuable contributions together with our own efforts in this area. Special mention must be made of Hagai Attias who is an invaluable collaborator. Hagai introduced us to probabilistic graphical models and Bayesian inference methods. He was an integral person in our fruitful collaboration, on which this book is based.

Many students, postdocs, fellows, and UCSF faculty members have collaborated with us over the years. These include Leighton Hinkley, David Wipf, Johanna Zumer, Julia Owen, Sarang Dalal, Isamu Kumihashi, Alex Herman, Naomi Kort, Ethan Brown, Rodney Gabriel, Maneesh Sahani, Adrian Guggisberg, Juan Martino, Kenneth Hild, Matthew Brookes, Carsten Stahlhut, Oleg Portniaguine, Erik Edwards, Ryan Canolty, David McGonigle, Tal Kenet, Theda Heinks-Maldonado, Aliu Sheye, Dameon Harrell, Josiah Ambrose, Sandeep Manyam, William McClay, Virginie van Wassenhove, Ilana Hairston, Maria Ventura, Zhao Zhu, Corby Dale, Tracy Luks, Kelly Westlake, Kamalini Ranasinghe, Karuna Subramanium, Carrie Niziolek, Zarinah Agnew, Carly Demopoulos, Megan Thomson, Peter Lin, Tulaya Limpiti, Lisa Dyson, Pew Puthividya, Jiucang Hao, Phiroz Tarapore, Dario Englot, Heidi Kirsch, Sophia Vinogradov, Michael Merzenich, Christoph Schreiner, Elizabeth Disbrow, Mitchel Berger, Edward Chang, Nick Barbaro, Roland Henry, Sarah Nelson, William Dillon, Jim Barkovich, Nancy Byl, David Blake, Keith Vossel, Elliot Sherr, Elysa Marco, Josh Wooley, Marilu Gorno-Tempini, Robert Knight, and 
Pratik Mukherjee. Also, we are deeply indebted to the following fantastic and incredibly dedicated staff at the Biomagnetic Imaging Laboratory: Susanne Honma, Mary Mantle, Anne Findlay-Dowling, Danielle Mizuiri, and Garrett Coleman.

Furthermore, several people have been particularly influential, and have greatly helped us to enhance our understanding of this field. Such people include Thomas Ferree, Mike X. Cohen, Stephen E. Robinson, Jiri Vrba, Guido Nolte, and Barry Van Veen. We would also like to thank Daniel Palomo for his effort in editing the manuscript of this book. Finally, we thank the following collaborators for their friendship and invaluable support to our work over nearly 20 years: John Houde, Steven Cheung, David Poeppel, Alec Marantz, and Tim Roberts.

We kindly ask readers to visit www.electromagneticbrainimaging.info. Supplementary information, as well as error corrections (if necessary), is uploaded to this website.

November 2014

Kensuke Sekihara

Srikantan S. Nagarajan 


\section{Contents}

1 Introduction to Electromagnetic Brain Imaging . . . . . . . . . 1

1.1 Functional Brain Imaging and Bioelectromagnetic

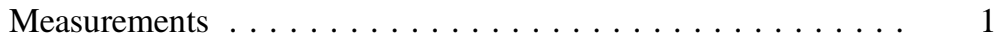

1.2 Sensing Magnetic Fields from the Brain . . . . . . . . . . 2

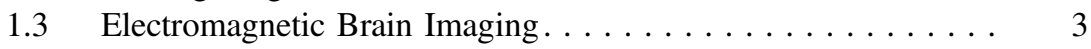

1.3.1 Forward Model. . . . . . . . . . . . . . . 3

1.3.2 Inverse Algorithms . . . . . . . . . . . . 4

1.4 From Source Imaging to Functional Connectivity Imaging . . . . 6

1.5 Examples of Clinical Applications . . . . . . . . . . 6

1.5.1 Functional Mapping for Preoperative Neurosurgical

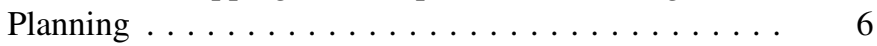

1.5.2 Functional Connectivity Imaging $\ldots \ldots \ldots \ldots \ldots \ldots$

References. . . . . . . . . . . . . . 8

2 Minimum-Norm-Based Source Imaging Algorithms . . . . . . . . 9

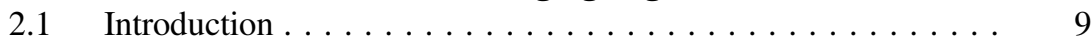

2.2 Definitions........................ 9

2.3 Sensor Lead Field. . . . . . . . . . . . . . . . 10

2.4 Voxel Source Model and Tomographic Source

2.5 Maximum Likelihood Principle and the Least-Squares

Method ......................... 13

2.6 Derivation of the Minimum-Norm Solution . . . . . . . . . 14

2.7 Properties of the Minimum-Norm Solution. . . . . . . . . . 15

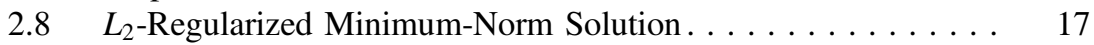

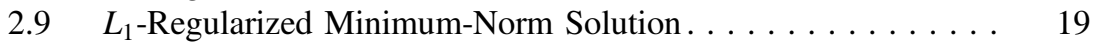

2.9.1 $L_{1}$-Norm Constraint. . . . . . . . . . . . . . . 19

2.9.2 Intuitive Explanation for Sparsity . . . . . . . . . 20

2.9.3 Problem with Source Orientation Estimation. . . . . . 23 
2.10 Bayesian Derivation of the Minimum-Norm Method . . . . . . . 24

2.10.1 Prior Probability Distribution and Cost Function. . . . . 24

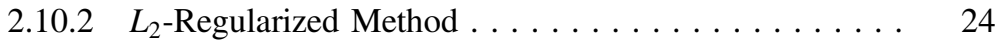

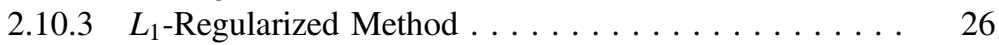

References. ....................... 28

3 Adaptive Beamformers . . . . . . . . . . . . . . . . . . . . . . . 29

3.1 Introduction and Basic Formulation. . . . . . . . . . . . . . . . 29

3.2 Classical Derivation of Adaptive Beamformers . . . . . . . . . 30

3.2.1 Minimum-Variance Beamformers with Unit-Gain

Constraint ................ 30

3.2.2 Minimum-Variance Beamformer with Array-Gain

Constraint .................. 31

3.2.3 Minimum-Variance Beamformer

with Unit-Noise-Gain Constraint. . . . . . . . . . . 32

3.3 Semi-Bayesian Derivation of Adaptive Beamformers. . . . . . . 33

3.4 Diagonal-Loading and Bayesian Beamformers . . . . . . . . . 35

3.5 Scalar Adaptive Beamformer with Unknown

Source Orientation . . . . . . . . . . . . . . 36

3.5.1 Expressions for the Unit-Gain Constraint

3.5.2 Expressions for the Array-Gain

3.6 Vector-Type Adaptive Beamformer. . . . . . . . . . . . . . . 38

3.6.1 Vector Beamformer Formulation. . . . . . . . . . . . . 38

3.6.2 Semi-Bayesian Formulation . . . . . . . . . . . . 40

3.7 Narrow-Band Beamformer . . . . . . . . . . . . . . . . . 42

3.7.1 Background ................... 42

3.7.2 Time-Domain Implementation . . . . . . . . . . . . . . 42

3.7.3 Frequency-Domain Implementation . . . . . . . . . . . . . . 43

3.7.4 Five-Dimensional Brain Imaging. . . . . . . . . . . . . . . . . . . . 44

3.8 Nonadaptive Spatial Filters. . . . . . . . . . . . . . . . . . 44

3.8.1 Minimum-Norm Filter . . . . . . . . . . . . . 44

3.8.2 Weight-Normalized Minimum-Norm Filter. . . . . . . 46

$3.8 .3 \quad$ sLORETA Filter. . . . . . . . . . . . . . . . 46

3.9 Recursive Null-Steering (RENS) Beamformer. . . . . . . . . 47

3.9.1 Beamformer Obtained Based on Beam-Response

Optimization .......................... 47

3.9.2 Derivation of RENS Beamformer . . . . . . . . . 48

References........................ 49

4 Sparse Bayesian (Champagne) Algorithm $\ldots \ldots \ldots \ldots \ldots \ldots$

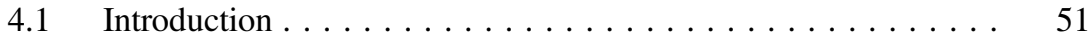

4.2 Probabilistic Model and Method Formulation ........... 52 
4.3 Cost Function for Marginal Likelihood Maximization . . . . . . . 54

$4.4 \quad$ Update Equations for $\alpha \ldots \ldots \ldots \ldots \ldots \ldots 6$

4.5 Modified Algorithm Integrating Interference Suppression. . . . . 58

4.6 Convexity-Based Algorithm . . . . . . . . . . . . . . . 59

4.6.1 Deriving an Alternative Cost Function. . . . . . . . . . 59

4.6.2 Update Equation for $z \ldots \ldots \ldots \ldots \ldots \ldots \ldots$

4.6 .3 Update Equation for $x_{k} \ldots \ldots \ldots \ldots \ldots \ldots \ldots$

4.6.4 Update Equation for $v \ldots \ldots \ldots \ldots \ldots \ldots \ldots \ldots \ldots$

4.6.5 Summary of the Convexity-Based Algorithm . . . . . . 62

4.7 The Origin of the Sparsity . . . . . . . . . . . . . . 63

4.8 Extension to Include Source Vector Estimation. . . . . . . . . . . . 65

4.8.1 Update Equation for $Z_{j} \ldots \ldots \ldots \ldots 6$

4.8.2 Update Equation for $s_{j}\left(t_{k}\right) \ldots \ldots \ldots \ldots \ldots$

$4.8 .3 \quad$ Update Equation for $\boldsymbol{\Upsilon}_{j} \ldots \ldots \ldots \ldots \ldots$

4.9 Source Vector Estimation Using Hyperparameter Tying. . . . . . 69

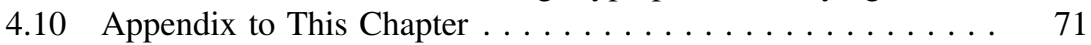

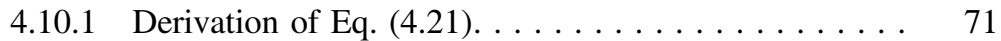

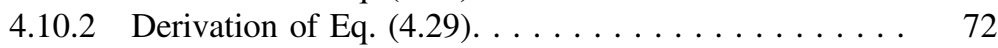

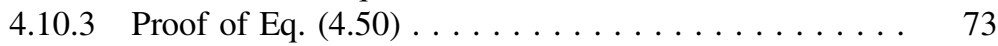

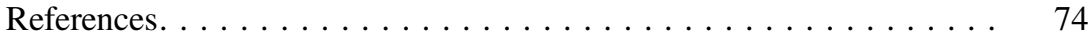

5 Bayesian Factor Analysis: A Versatile Framework

for Denoising, Interference Suppression,

and Source Localization . . . . . . . . . . . . . . . . . . . . . 75

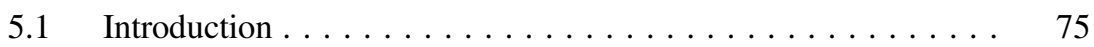

5.2 Bayesian Factor Analysis. . . . . . . . . . . . . 75

5.2.1 Factor Analysis Model . . . . . . . . . . . 75

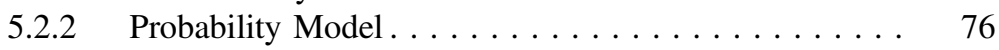

$5.2 .3 \quad$ EM Algorithm . . . . . . . . . . . . . . 77

5.2.4 Computation of Marginal Likelihood. . . . . . . . . 79

5.2.5 Summary of the BFA Algorithm. . . . . . . . . 81

5.3 Variational Bayes Factor Analysis (VBFA) . . . . . . . . 82

5.3.1 Prior Distribution for Mixing Matrix . . . . . . . . 82

5.3.2 Variational Bayes EM Algorithm (VBEM). . . . . . . 84

5.3 .3 Computation of Free Energy . . . . . . . . . . 92

5.3.4 Summary of the VBFA Algorithm . . . . . . . . . . . . . 95

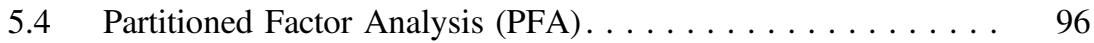

$5.4 .1 \quad$ Factor Analysis Model . . . . . . . . . . . . . . . 96

5.4 .2 Probability Model . . . . . . . . . . . . . . . 97

5.4.3 VBEM Algorithm for PFA. . . . . . . . . . . . 97

5.4 .4 Summary of the PFA Algorithm . . . . . . . . . . . 100

5.5 Saketini: Source Localization Algorithm Based

on the VBFA Model. . . . . . . . . . . . . . . . . 101

$5.5 .1 \quad$ Data Model . . . . . . . . . . . . . . . . 101 
5.5.2 Probability Model. . . . . . . . . . . . . . . . 102

5.5 .3 VBEM Algorithm. . . . . . . . . . . . . . . 103

5.5.4 Summary of the Saketini Algorithm . . . . . . . . . . 107

5.6 Numerical Examples . . . . . . . . . . . . . . . . . . . . . . . 107

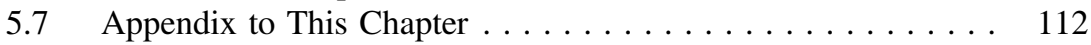

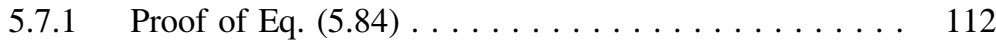

5.7.2 Proof of Eq. (5.94) . . . . . . . . . . . . . . 114

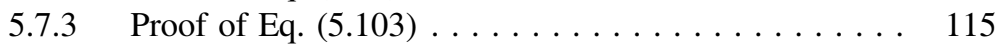

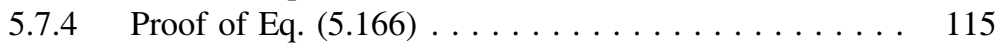

References. . . . . . . . . . . . . . . . . . . . . 117

6 A Unified Bayesian Framework for MEG/EEG Source

Imaging . . . . . . . . . . . . . . . . . . . . . . . . . . . 119

$6.1 \quad$ Introduction . . . . . . . . . . . . . . . . . . . . 119

6.2 Bayesian Modeling Framework. . . . . . . . . . . . 121

6.3 Bayesian Modeling Using General Gaussian Scale

Mixtures and Arbitrary Covariance Components . . . . . . . . . . 122

6.3.1 The Generative Model. . . . . . . . . . . . . . . . 122

6.3.2 Estimation and Inference . . . . . . . . . . . 123

6.3.3 Source MAP or Penalized Likelihood Methods. . . . . 127

6.3.4 Variational Bayesian Approximation . . . . . . . . . . 131

6.4 Selection of Covariance Components $C \ldots \ldots \ldots \ldots \ldots \ldots . . \ldots 133$

6.5 Discussion . . . . . . . . . . . . . . . . . . . . . 134

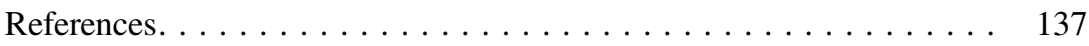

\section{Source-Space Connectivity Analysis Using Imaginary}

Coherence . . . . . . . . . . . . . . . . . . . . . . . . . . . . . . . 139

$7.1 \quad$ Introduction . . . . . . . . . . . . . . . . . . . . . . . . . . . . . 139

7.2 Source-Space Coherence Imaging . . . . . . . . . . . . . 140

7.3 Real and Imaginary Parts of Coherence . . . . . . . . . . . . 141

7.4 Effects of the Leakage. . . . . . . . . . . . . . . . . . . . . 143

7.4.1 Leakage Effects on the Magnitude Coherence. . . . . . 143

7.4.2 Leakage Effects on the Imaginary Coherence . . . . . . . 144

7.5 Corrected Imaginary Coherence . . . . . . . . . . . . . . . . 145

7.5.1 Modification of Imaginary Coherence . . . . . . . . . . 145

7.5.2 Factorization of Mutual Information . . . . . . . . . 146

7.5.3 Residual Coherence. . . . . . . . . . . . . . . . . . 148

7.5.4 Phase Dependence of the Corrected Imaginary

Coherences .................... 150

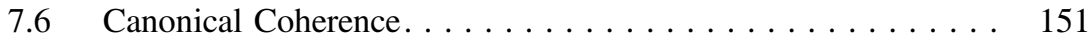

7.6.1 Canonical Magnitude Coherence. . . . . . . . . . . . . 151

7.6.2 Canonical Imaginary Coherence . . . . . . . . . . . . 154

7.6.3 Canonical Residual Coherence . . . . . . . . . . . . . 157 
7.6.4 Computing Coherence When Each Voxel

has Multiple Time Courses . . . . . . . . . . . . . 158

7.7 Envelope Correlation and Related Connectivity Metrics . . . . . 159

7.7.1 Envelope Correlation. . . . . . . . . . . . . . . . . 159

7.7.2 Residual Envelope Correlation . . . . . . . . . . . . . . . . . . . . . . . . 160

7.7.3 Envelope Coherence . . . . . . . . . . . . . . . . . 160

7.8 Statistical Thresholding of Coherence Images . . . . . . . . . . . . . . . . 161

7.9 Mean Imaginary Coherence (MIC) Mapping . . . . . . . . . . . . . . . . . 162

7.10 Numerical Examples . . . . . . . . . . . . . . . . . . . . . . . . . . . . . . . . . . . . 163

References. . . . . . . . . . . . . . . . . . . . . . . 168

\section{Estimation of Causal Networks: Source-Space Causality}

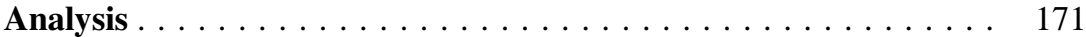

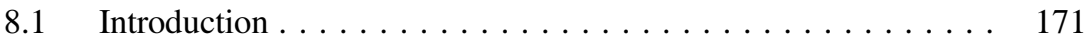

8.2 Multivariate Vector Autoregressive (MVAR) Process . . . . . 171

8.2.1 MVAR Modeling of Time Series . . . . . . . . . . . 171

8.2.2 Coherence and Partial Coherence of the MVAR Process . . . . . . . . . . . . . . . . . . . 173

8.3 Time-Domain Granger Causality. . . . . . . . . . . . . . . . 174

8.3.1 Granger Causality for a Bivariate Process . . . . . . . . 174

8.3.2 Multivariate Granger Causality . . . . . . . . . . . . . . 175

8.3 .3 Total Interdependence . . . . . . . . . . . . . . . . . 177

8.4 Spectral Granger Causality: Geweke Measures . . . . . . . . . . . 178

8.4.1 Basic Relationships in the Frequency Domain . . . . . 178

8.4.2 Total Interdependence and Coherence . . . . . . . . . . 179

8.4.3 Deriving Causal Relationships in the Frequency Domain . . . . . . . . . . . . . . . . . . 180

8.5 Other MVAR-Modeling-Based Measures . . . . . . . . . . . . . . . . . 182

8.5.1 Directed Transfer Function (DTF) . . . . . . . . . . 182

8.5.2 Relationship Between DTF and Coherence. . . . . . . . 183

8.5.3 Partial Directed Coherence (PDC). . . . . . . . . . . . 184

8.6 Transfer Entropy. . . . . . . . . . . . . . . . . . . . 185

8.6 .1 Definition . . . . . . . . . . . . . . . 185

8.6.2 Transfer Entropy Under Gaussianity Assumption . . . . 186

8.6.3 Equivalence Between Transfer Entropy and Granger Causality . . . . . . . . . . . . . . . . 187

8.6.4 Computation of Transfer Entropy . . . . . . . . . . . . 188

8.7 Estimation of MVAR Coefficients . . . . . . . . . . . . . . . 190

8.7.1 Least-Squares Algorithm . . . . . . . . . . . . . . . . . 190

8.7.2 Sparse Bayesian (Champagne) Algorithm. . . . . . . . . 191

$8.8 \quad$ Numerical Examples . . . . . . . . . . . . . . . . . . . . . . . . . . . . 192

8.8.1 Experiments Using Bivariate Causal Time Series . . . . 192

8.8.2 Experiments Using Trivariate Causal Time Series . . . 194

References. . . . . . . . . . . . . . . . . . . . . . . . . . 198 
9 Detection of Phase-Amplitude Coupling in MEG Source Space:

An Empirical Study . . . . . . . . . . . . . . . . . . . . . . . . . . . 199

9.1 Introduction . . . . . . . . . . . . . . . . . . . . . . . . 199

9.2 Types of Cross-Frequency Coupling . . . . . . . . . . . . . . 200

9.3 Local PAC and Cross-Location PAC . . . . . . . . . . . . 201

9.4 Quantification of Phase-Amplitude Coupling . . . . . . . . . . . 202

9.4.1 Instantaneous Amplitude and Phase. . . . . . . . . . 202

9.4.2 Amplitude-Phase Diagram. . . . . . . . . . . . . . 202

9.4 .3 Modulation Index $(\mathrm{MI}) \ldots \ldots \ldots . \ldots \ldots 203$

9.4.4 Phase-Informed Time-Frequency Map . . . . . . . . . . . . 204

9.5 Source Space PAC Analysis: An Example Study

Using Hand-Motor MEG Data . . . . . . . . . . . . . . . . . . 204

9.5.1 Experimental Design and Recordings . . . . . . . . . 204

9.5.2 Data Analysis. . . . . . . . . . . . . . . . . . 204

9.5.3 Results of Local PAC Analysis. . . . . . . . . . . . . 206

9.5.4 Results of Analyzing Cross-Location PACs . . . . . . . 209

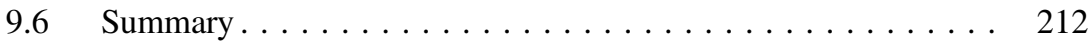

References......................... 212

Appendix A: Bioelectromagnetic Forward Modeling . . . . . . . . . . . 215

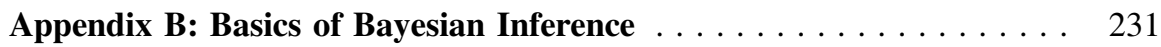

Appendix C: Supplementary Mathematical Arguments . . . . . . . . . . 247

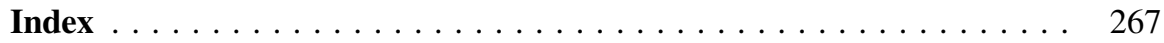

